\title{
Safety distance in the resection of colorectal lung metastases: A prospective evaluation of satellite tumor cells with immunohistochemistry
}

\author{
Stefan Welter, MD, ${ }^{\mathrm{a}}$ Dirk Theegarten, MD, ${ }^{\mathrm{b}}$ Tanja Trarbach, MD,${ }^{\mathrm{c}}$ Frank Maletzki, ScD, ${ }^{\mathrm{d}}$ \\ Georgios Stamatis, MD, ${ }^{a}$ and Martin Tötsch, MD $^{\mathrm{b}}$
}

\begin{abstract}
Objective: After complete pulmonary metastasectomy of colorectal lung metastases, tumor recurrence at the site of former resection develops in some patients. Well-described risk factors for local recurrence in the lung include incomplete resection and aerogenous spread of floating cancer cell clusters. The aim of this study was to describe the distribution and frequency of satellite cancer cells or clusters in resected tissue to deduce recommendations for safety margins in the future.
\end{abstract}

\begin{abstract}
Methods: Seventeen colorectal metastases from 10 patients were processed. Evaluation for pattern of growth, satellite cancer cells, and inflammatory reaction at the surface of the metastases was performed on 102 sections with CDX2 and cytokeratin 20 stains. The distance between the surface of the nodule and the satellite cancer cell was measured for each satellite and statistically evaluated.
\end{abstract}

Results: As a pattern of growth, interstitial spread, inflammatory reaction, and lymphangitic spread were observed in $41.2 \%, 35.3 \%$, and $23.5 \%$, respectively. A total of 205 satellite cancer cells were identified in 16 of 17 metastases with a mean distance to the nodule of $0.99 \pm 0.85 \mathrm{~mm}$ (range, $0.06-6.43 \mathrm{~mm}$ ). The percentages of satellite tumor cells that are likely to be found within $1.59 \mathrm{~mm}, 3.43 \mathrm{~mm}$, and $7.4 \mathrm{~mm}$ around the nodule are $68.27 \%, 95.5 \%$, and $99.73 \%$, respectively.

Conclusions: Satellite tumor cells, a potential source for local recurrence, are found in a high number around colorectal lung metastases. As a standard of care, a safety distance of $3 \mathrm{~mm}$ for small metastases and 8 to $10 \mathrm{~mm}$ for larger metastases must be maintained around the lesion to prevent local recurrence. (J Thorac Cardiovasc Surg 2011;141:1218-22)

Surgery for pulmonary metastases from colorectal cancer is a well-established method, but there is a low probability for cure. After a complete pulmonary metastasectomy, up to $75 \%$ of patients have tumor recurrences, with most of them occurring at other sites. ${ }^{1}$ Nevertheless, pulmonary recurrence at the site of the former resection develops in some patients. This is the sole area that can be influenced by the surgeon with an adequate margin. The known reasons for local recurrence in the lung are incomplete resection, lymphangitic spread, and floating cancer cells around the metastases, ${ }^{2,3}$ but even when the pathologist certifies a complete resection, local recurrence of the cancer may occur. An explanation of local recurrence after microscopically

\footnotetext{
From the Department of Thoracic Surgery, ${ }^{\mathrm{a}}$ Ruhrlandklinik Essen, Essen, Germany; Institute of Pathology and Neuropathology, ${ }^{\mathrm{b}}$ University-Hospital Essen, University of Duisburg-Essen, Essen, Germany; Department of Medical Oncology, ${ }^{\mathrm{c}}$ West German Cancer Center, University of Duisburg-Essen, Essen, Germany; and MedService, ${ }^{\mathrm{d}}$ Department of Biostatistics, Essen, Germany.

Disclosures: Authors have nothing to disclose with regard to commercial support.

Received for publication March 29, 2010; revisions received June 30, 2010; accepted for publication Aug 1, 2010; available ahead of print Dec 16, 2010.

Address for reprints: Stefan Welter, MD, Ruhrlandklinik Thoracic Surgery, Tüschener Weg 40, 45239 Essen, Germany (E-mail: stefan.welter@ ruhrlandklinik.uk-essen.de).

0022-5223/\$36.00

Copyright (C) 2011 by The American Association for Thoracic Surgery doi:10.1016/j.jtcvs.2010.08.089
}

complete resection may be incomplete removal of satellite tumor cells during the operation. The distance of such satellite cells from the original metastasis has not been further examined yet.

To prevent local recurrence in the lung, Rusch ${ }^{4}$ recommended a safety distance of 0.5 to $1 \mathrm{~cm}$ for pulmonary metastasectomy. Rolle and colleagues ${ }^{5}$ reported no local recurrences after laser resection of multiple metastases in 328 patients. This statement was not further verified because a regular follow-up of those patients for local recurrence was not completed. However, laser resection is well known for leaving a 5-mm band of carbonized lung tissue at the resection site that is similar to a safety margin. We conducted this study to investigate the extension of tumor cell spread and to conclude the best operative technique for pulmonary metastasectomy. More specifically, the aim of this study was to evaluate the distance and the frequency of single tumor cells or tumor cell aggregates around colorectal lung metastases to deduce recommendations for a safety margin in the future.

\section{MATERIALS AND METHODS}

From October 2007 to March 2009, metastases from 10 patients with a history of colorectal cancer were collected. From each patient, 1 to 3 metastases were investigated. The hospital review board waived the need for 


\section{Abbreviations and Acronyms \\ $\mathrm{ASFC}=$ aerogenous spread with floating cancer cell clusters \\ $\mathrm{CK}=$ cytokeratin \\ $\mathrm{SD}=$ standard deviation}

patient consent because the day-long procedures for specimen preparation were only intensified but not principally changed. Further, no patientspecific data were evaluated. When an adenocarcinoma was detected by histology, the tumor was defined as colorectal metastasis when the following tumor characteristics were present in immunohistochemistry: positive reaction for the homeobox protein CDX2 or cytokeratin (CK)20 and negative reaction for thyroid transcription factor-1 or CK7.

\section{Sample Preparation}

One to 3 lung specimens from each patient were injected and fixed with $10 \%$ formalin without sticking the needle into the tumor until the alveolar lung tissue had the approximate size of inflated normal lung tissue. After a minimal incubation time of 8 hours, the specimens were dried with a cellulose cloth, measured, and characterized. If present, a staple line was removed with a pair of scissors, and $3 \mathrm{~mm}$ of safety distance was added in the pathology report. The resection area was swabbed with Indian ink, and the specimen was cut into 3-mm slices beginning on the side with the thickest part of lung tissue covering the metastasis. After each cut, the knife was cleaned under running water and dried with a clean paper towel so that potential tumor cell contamination of the knife blade was minimized. The largest diameter of the metastasis was noted, and up to 6 slices from 1 metastasis containing the border between the tumor and the normal lung tissue were embedded in paraffin. Sections of approximately $5 \mu \mathrm{m}$ were cut from each tissue block and stained with hematoxylin-eosin. To establish the correct diagnosis for each patient, immunohistochemistry analysis was performed using monoclonal antibodies against thyroid transcription factor-1, CDX2, and, if necessary, CK7 and CK20 (DAKO, Hamburg, Germany). Two sections from each paraffin bloc containing tumor tissue were prepared, one with CDX2 and one with CK20, as previously described. ${ }^{6}$

\section{Evaluation of Satellite Nodules}

Sections stained with hematoxylin-eosin, CDX2, and CK20 were examined with the Nikon Eclipse 80i microscope (Nikon Instruments Inc, Tokyo, Japan) for the presence of a pseudocapsule, interstitial growth, lymphangitic spread, satellite nodules, and aerogenous spread with floating cancer cell clusters (ASFC). ${ }^{3}$ The term "interstitial growth" is used for a tumor that grows into the alveolar septae with a tongue-like shape instead of forming a pseudocapsule. Single cells or cell clusters in the alveolar tissue that stained in an equivalent level to the main metastasis were defined as tumor satellites. Tumor cell clusters lying free in the alveolar space were defined as ASFC. The exact distance to the surface of the metastasis was measured for each satellite nodule, ASFC, or satellite cell using a personal computer and the NIS Elements 2.3 software (Nikon Instruments Inc). Interesting abnormalities observed in the sections were also noted.

\section{Statistical Analysis}

Data were analyzed with the Statistical Package for the Social Sciences 15.0 program for Windows (SPSS Inc, Chicago, Ill). The 1-sample Kolmogorov-Smirnov test was used to test for normal distribution of the distance data. The correlation between the diameter of metastases and the distance of tumor satellites from the metastases was evaluated by Pearson's correlation coefficient.
TABLE 1. Characteristics of 17 colorectal lung metastases

\begin{tabular}{lcr}
\hline \multicolumn{1}{c}{ Diameter $(\mathbf{m m})$} & Mean 23.3 & Median 17(2-65) \\
\hline Interstitial growth & $47 / 102$ sections & $7 / 17$ metastases $(41.2 \%)$ \\
Inflammatory infiltration & $32 / 102$ sections & $6 / 17$ metastases $(35.3 \%)$ \\
Lymphangitic spread & $4 / 102$ sections & $4 / 17$ metastases $(23.5 \%)$ \\
Peri/intravascular growth & $2 / 102$ sections & $2 / 17$ metastases $(11.8 \%)$ \\
Satellite tumor cells & 205 in 59/102 & $16 / 17$ metastases $(94.1 \%)$ \\
& sections & \\
Distance of satellite & Mean 0.99 & Median $0.72(0.06-6.43)$ \\
$\quad$ & & \\
\hline
\end{tabular}

\section{RESULTS}

In this study, 17 colorectal lung metastases from 10 patients were investigated, and 51 paraffin blocks and 102 sections were prepared for immunohistochemistry of CDX2 and CK20. The mean diameter of the metastases was $23.3 \mathrm{~mm}$, and the median was $17 \mathrm{~mm}$ with the diameters ranging from 2 to $65 \mathrm{~mm}$.

The formation of a surrounding pseudocapsule that consisted of compressed alveolar structures with variable thicknesses around the whole metastasis was seen in only 2 of 17 nodules $(11.8 \%)$. All other specimens had incomplete or, more often, no pseudocapsule at all, and some satellite tumor cells were found despite the formation of a pseudocapsule. Interstitial growth that made the border of the metastasis ill defined was found in 9 metastases; interstitial growth was observed in 47 of the total 102 sections $(52.9 \%)$. The remaining 55 sections from the other 8 metastases had a clearly defined surface of the nodule. Only 4 sections from 4 metastases $(23.5 \%)$ revealed a lymphangitic spread (Table 1). Satellite tumor cells were found in 14 of the 17 metastases $(82.4 \%)$ ). In 6 metastases $(35.3 \%)$, a relevant inflammatory reaction around the nodule was observed in 32 of 102 sections. In these cases many single tumor cells that were probably unhinged by lytic enzymes were found beyond the metastatic surface, and these cells were not considered for evaluation.

The ASFC, lymphangitic spread, and interstitial growth were identified by hematoxylin-eosin stains. Evaluation of the sections with immunohistochemical stains allowed the identification of more single tumor cells and small accumulations of cancer cells distant to the metastasis (Figure 1). We observed these satellite tumor cells floating almost exclusively in the alveolar space, which discriminates colorectal metastases from sarcoma metastases where satellite nodules are lying in the alveolar septae. Altogether, 205 satellites, either ASFC or distant single cells, were counted. The distance of the satellites from the surface of the metastasis was measured (Figure 2). The median distance was $0.72 \mathrm{~mm}$ (range, $0.06-6.43 \mathrm{~mm}$ ), and the mean of the distances was $0.99 \pm 0.85 \mathrm{~mm}$. Only 2 values $(6.43$ and $5.04 \mathrm{~mm})$ were more than $5 \mathrm{~mm}$, and 6 of the 205 distances exceeded $3 \mathrm{~mm}$. The 1-sample 


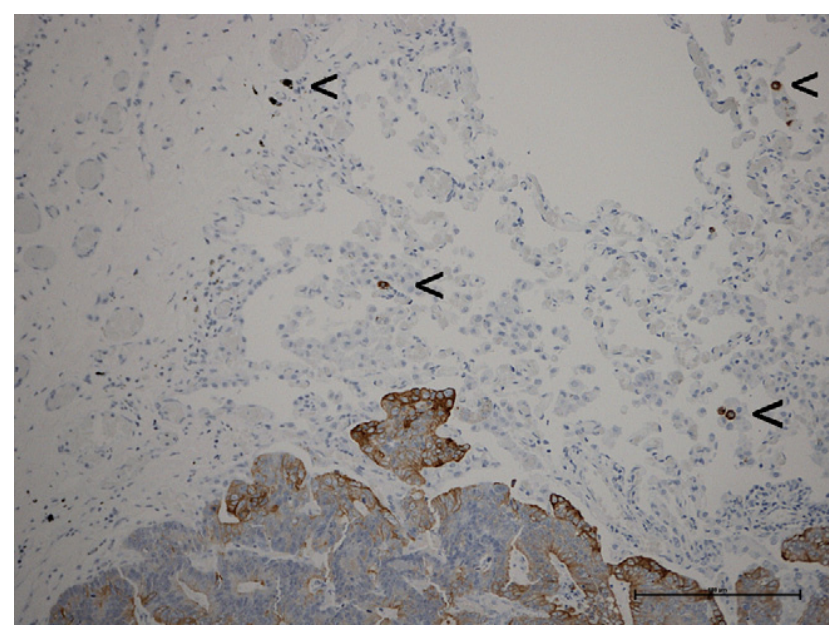

FIGURE 1. Colorectal lung metastases as shown by CK20 staining with black arrows on the satellite tumor cells ( $\times 10$ magnification).

Kolmogorov-Smirnov test showed that the observed distribution of the distance data was significantly different from the normal distribution $(P<.001)$. Calculating the natural logarithm of the distance data resulted in a log-normal data set (Kolmogorov-Smirnov test: $P=.776$ ) (Figure 3).

The probability of complete removal of satellite tumor cells within a given distance was evaluated from the probability density function of the normal distribution using the mean and standard deviation (SD) of the log normal distance data as parameters.

Within a distance of $1.6 \mathrm{~mm}$ (mean $+1 \mathrm{SD}), 3.4 \mathrm{~mm}$ (mean $+2 \mathrm{SD})$, and $7.4 \mathrm{~mm}($ mean $+3 \mathrm{SD})$, the calculated probabilities were $68.3 \%, 95.5 \%$, and $99.7 \%$, respectively.

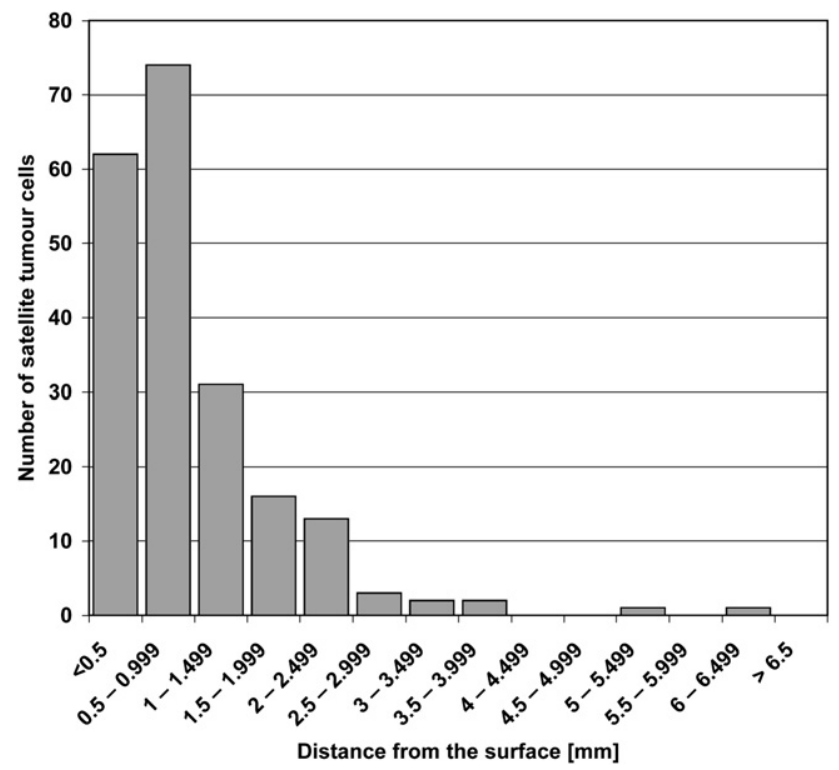

FIGURE 2. Distribution of tumor satellite cells beyond the surface of the metastases.

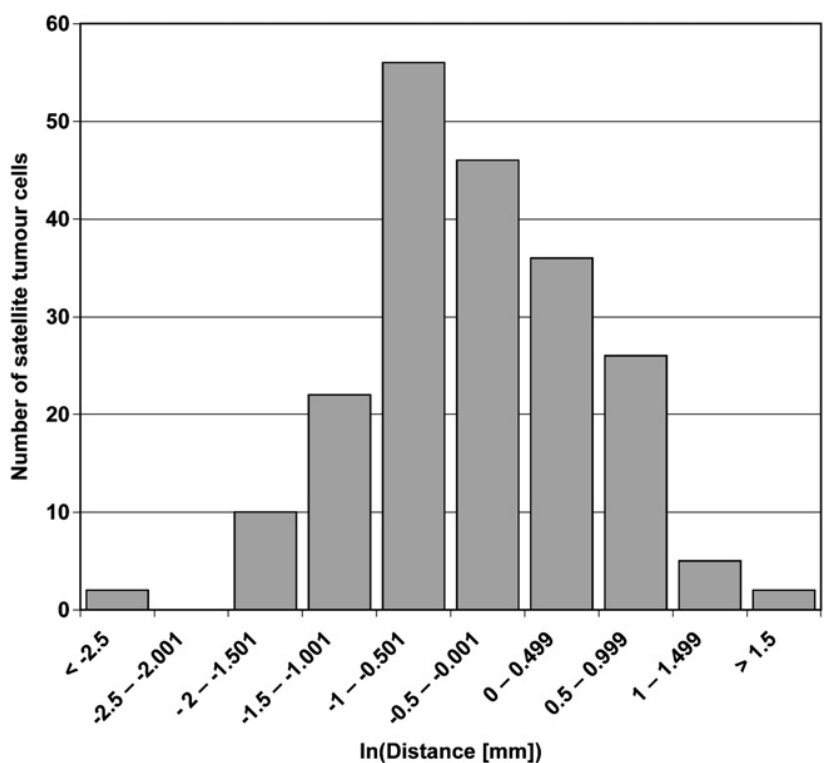

FIGURE 3. Distribution of the natural logarithms of the satellite tumor cell distances from the surface of the metastases.

A probability of $99.99 \%$ (mean $+4 \mathrm{SD}$ ) corresponded to a distance of $15.9 \mathrm{~mm}$ (Figure 4).

The Pearson's correlation coefficient (R) for the correlation of the nodule diameter and the distance of satellite tumor cells from the metastases was $\mathrm{R}=0.101$, which was not statistically significant $(P=.149)$. This implicates that a correlation between the nodule diameter and the distance of satellite tumor cells from the metastases was not demonstrated, even though metastases smaller than $2 \mathrm{~cm}$ did not have satellite tumor cells further than $3 \mathrm{~mm}$ from the surface and the regression line was gently inclined (Figure 5).

\section{DISCUSSION}

Colon cancer is one of the leading causes of cancer death in western countries. After initial diagnosis, more than two thirds of these patients undergo primary curative resection. Distant metastases are usually the cause of tumor recurrence. $^{7}$ Despite having stage IV disease, some highly selected patients with only a few pulmonary metastases can undergo surgical metastasectomy and still have a curative option with a 5-year survival up to $60 \%$. $^{3}$ The results of 5206 cases assessed in the International Registry of Lung Metastases indicated that lung metastasectomy, in general, is a safe and potentially curative procedure. ${ }^{8}$ This database included 645 colorectal pulmonary metastases. With regard to all epithelial tumors in this database, recurrences $(44 \%$ intrathoracic, $56 \%$ extrathoracic) were diagnosed in $46 \%$, and $28 \%$ of these patients had recurrent pulmonary metastasectomies. Even higher numbers of distant recurrences within 9 months of resection $(60 \%$, two thirds intrapulmonary) were found by Rolle and colleagues ${ }^{5}$ and Welter and 


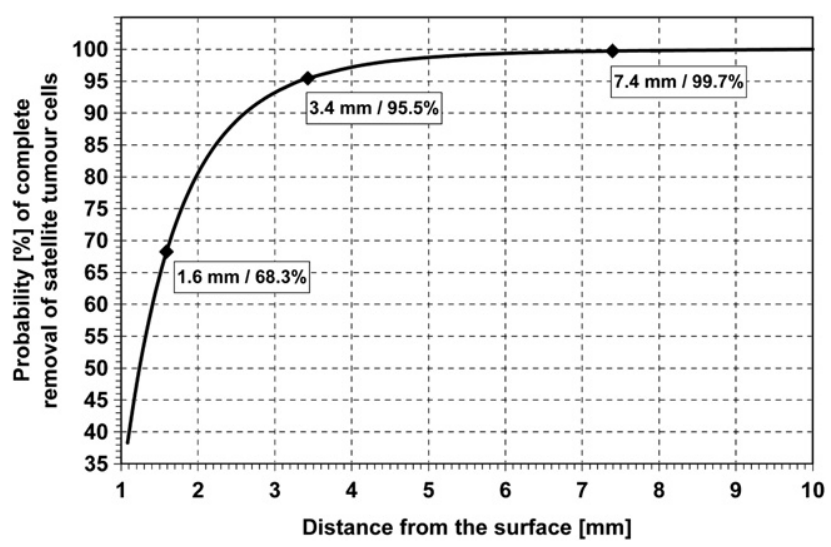

FIGURE 4. Distribution of the probability for a complete removal of satellite tumor cells depending on the distance from the surface of the metastasis.

colleagues $(75 \%) .{ }^{1}$ New metastases and a local recurrence at a former resection site may be reasons for intrapulmonary tumor recurrence. Microscopically incomplete resection was identified as a risk factor resulting in 8 local recurrences at 11 sites with an $\mathrm{R} 1$ resection, but local recurrence in the lung occurred in 14 of 22 cases even when microscopically R0 resection was confirmed. ${ }^{2}$ Main factors associated with early relapse were identified as histologic type of tumor (more relapses in sarcoma and less in adenocarcinoma), a short disease-free interval between primary tumor resection and lung metastases, and the number of metastases. ${ }^{9}$ Shiono and colleagues ${ }^{3}$ published a report on risk factors for locoregional recurrence in the lung. They found that both microscopically incomplete resections and the presence of more than 10 ASFC per metastasis were associated with local recurrence, and that ASFC $(P=.004)$, vascular invasion $(P=.002)$, pleural invasion $(P=.037)$, and lymphangitic spread $(P=.032)$ were significant prognostic factors. When the colorectal metastases contained ASFC and vascular invasion, the 5-year survival of these patients was only $24.7 \%$, but when the patients did not have these factors, the 5-year survival was $93.3 \%(P=.0002)$. These results were verified by others demonstrating that the radicality of resection with clear margins had a significant impact on survival. ${ }^{10,11}$

To prevent local recurrence, Rusch ${ }^{4}$ advised to remove a conical-shaped wedge of pulmonary parenchyma circumferentially around the nodule and to take a $0.5-$ to $1.0-\mathrm{cm}$ margin of normal lung tissue with it in all directions. This recommendation was based on a case series with local recurrences. A similar rule is well known for hepatic colorectal metastases, but a recent publication demonstrated that even a minimal distance of $1 \mathrm{~mm}$ can be sufficient. ${ }^{12}$

The minimal safety distance to reach clear margins in the lung is still unclear. Higashiyama and colleagues ${ }^{13}$ found that the lavage cytology of surgical margins is associated with an elevated local recurrence rate when tumor cells

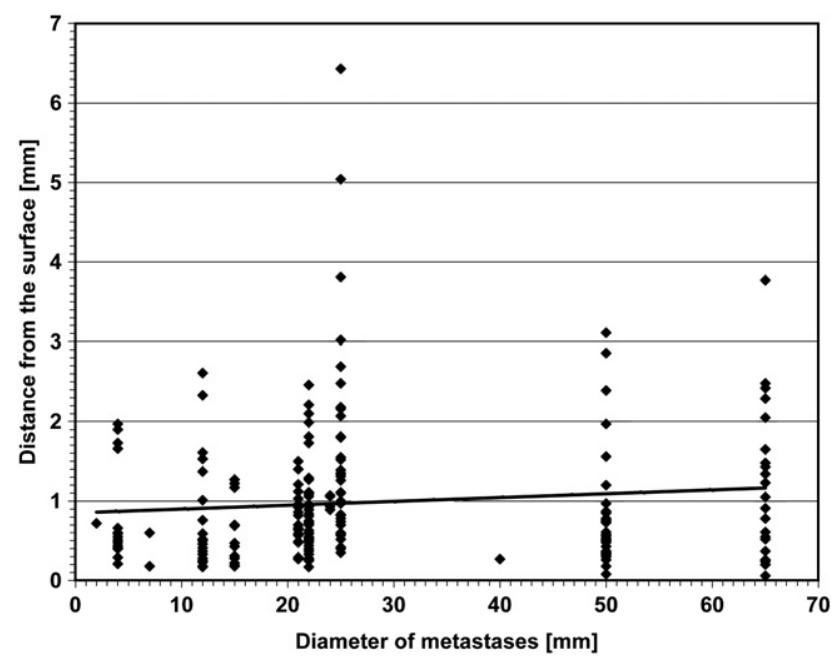

FIGURE 5. Scatter plot with the distances of satellite tumor cells and the diameter of the metastasis. The Pearson's correlation coefficient $(\mathrm{R}=0.101)$ between the 2 attributes is not statistically significant $(P=.149)$. The equation for the regression line is distance $(\mathrm{mm})=0.0048 \times$ diameter of the metastasis $(\mathrm{mm})+0.8507$.

were found. Potentially invisible tumor cells have been found by molecular margin analysis and were associated with a higher local recurrence rate after resection of lung cancer. ${ }^{14}$ Together, these publications emphasize that tumor cells beyond the surface of the metastases may be the origin of local recurrences.

The purpose of our study was to define the necessary resection safety distances around colorectal lung metastases. The local pattern of growth was described, and the distance of satellite tumor cells from the surface of the metastasis was then measured. We demonstrated that most $(95 \%)$ of the satellite tumor cells were in a range 0 to $3.5 \mathrm{~mm}$ from the surface of the metastasis, but some of the cells were found more than $5 \mathrm{~mm}$ from the surface. Furthermore, we demonstrated that the distance of satellite tumor cells in smaller metastases $(<2 \mathrm{~cm})$ did not exceed $3 \mathrm{~mm}$, even though a statistically significant correlation between the distance of satellite cells and the diameter of the metastases was not found. On the basis of these results, we propose that a safety distance of 8 to $10 \mathrm{~mm}$ is necessary for lesions larger than $2 \mathrm{~cm}$.

\section{Limitations}

This study has several limitations. The immunohistochemical coloring of the tumors was not homogenous in all metastases, so the number of counted ASFC and satellite cells may have been underestimated. Therefore, the likelihood for a complete resection with a defined safety margin may have been even lower. It was also impossible to distinguish between satellite nodules or cells coming from the metastases and satellites composed of hematogenously spread tumor cells from the primary tumor. However, the 
distribution of the satellite tumor cells in this study (Figure 2) suggests that these cells originate from the metastatic nodule and are not new micrometastases from hematogenous spread, and larger safety margins would not be able to solve the latter problem. Furthermore, this study was limited to a small number of patients with only a limited number of metastases from each patient to be prepared and examined. Nevertheless, these limitations should not disrupt the message that safety margins are important and that a 5-mm distance is not enough to avoid residual tumor cells in larger metastases, which may be responsible for local recurrences.

\section{CONCLUSIONS}

Satellite tumor cells may be found circular around colorectal metastases, with most of them residing in alveolar spaces. The majority of the satellite tumor cells can only be identified with immunohistochemistry. These cells are potentially the source for recurrent tumor growth. To prevent local recurrence, a safety distance of 8 to $10 \mathrm{~mm}$ for larger metastases must be maintained around the lesion.

\section{References}

1. Welter S, Jacobs J, Krbek T, Poettgen C, Stamatis G. Prognostic impact of lymph node involvement in pulmonary metastases from colorectal cancer. Eur J Cardiothorac Surg. 2007;31:167-72.

2. Welter S, Jacobs J, Krbek T, Krebs B, Stamatis G. Long-term survival after repeated resection of pulmonary metastases from colorectal cancer. Ann Thorac Surg. 2007;84:203-10.
3. Shiono S, Ishii G, Nagai K, Yoshida J, Nishimura M, Murata Y, et al. Histopathologic prognostic factors in resected colorectal lung metastases. Ann Thorac Surg. 2005;79:278-82.

4. Rusch VW. Pulmonary metastasectomy. Current indications. Chest. 1995;107 (6 Suppl):322-31.

5. Rolle A, Pereszlenyi A, Koch R, Richard M, Baier B. Is surgery for multiple lung metastases reasonable? A total of 328 consecutive patients with multiple laser metastasectomies with a new 1318-nm Nd:YAG laser. J Thorac Cardiovasc Surg. 2006;131:1236-42.

6. Tanaka S, Saito K, Ito T, Tajima K, Mogi A, Shitara Y, et al. CDX2 as a useful marker of colorectal adenocarcinoma metastases to lung in pre-operative biopsy specimens. Oncol Rep. 2007;18:87-92.

7. Hermanek P, Wiebelt H, Riedel S, Staimmer D, Hermanek P. Long term results of surgical therapy of colon cancer. Results of the Colorectal Cancer Study Group. Chirurg. 1994;65:287-97.

8. Pastorino U, Buyse M, Friedel G, Ginsberg RJ, Girard P, Goldstraw P, et al. Longterm results of lung metastasectomy: prognostic analyses based on 5206 cases. J Thorac Cardiovasc Surg. 1997;113:37-49.

9. Rios A, Galindo PJ, Torres J, Roca MJ, Robles R, Luján JA, et al. Factors causing early relapse after lung metastasis surgery. Eur J Cancer Care. 2007;16:26-32.

10. Pfannschmidt J, Dienemann H, Hoffmann H. Surgical resection of pulmonary metastases from colorectal cancer: a systematic review of published series. Ann Thorac Surg. 2007;84:324-38.

11. Vogelsang H, Haas S, Hierholzer C, Berger U, Siewert JR, Präuer H. Factors influencing survival after resection of pulmonary metastases from colorectal cancer. Br J Surg. 2004;91:1066-71.

12. Vandeweyer D, Neo EL, Chen JW, Maddern GJ, Wilson TG, Padbury RT. Influence of resection margins on survival in hepatic resections for colorectal liver metastases. HPB (Oxford). 2009;11:499-504.

13. Higashiyama M, Kodama K, Takami K, Higaki N, Yokouchi H, Nakayama T, et al. Intraoperative lavage cytologic analysis of surgical margins as a predictor of local recurrence in pulmonary metastasectomy. Arch Surg. 2002;137: 469-74.

14. Masasyesva BG, Tong BC, Brock MV, Pilkington T, Goldenberg D, Sidransky D, et al. Molecular margin analysis predicts local recurrence after sublobar resection of lung cancer. Int J Cancer. 2005;113:1022-5. 